\title{
Diacronie
}

Studi di Storia Contemporanea

$\mathrm{N}^{\circ} 12,4 \mid 2012$

Sulle tracce delle idee

\section{Monfalcone, storia di un cantiere navale}

\section{Stefania Elena Carnemolla}

\section{(2) OpenEdition}

\section{Journals}

\section{Edizione digitale}

URL: http://journals.openedition.org/diacronie/2584

DOI: 10.4000/diacronie.2584

ISSN: 2038-0925

\section{Editore}

Association culturelle Diacronie

Notizia bibliografica digitale

Stefania Elena Carnemolla, « Monfalcone, storia di un cantiere navale », Diacronie [Online], № 12, 4 ।

2012, documento 12, Messo online il 29 décembre 2012, consultato il 11 juin 2020. URL : http://

journals.openedition.org/diacronie/2584; DOI : https://doi.org/10.4000/diacronie.2584 


\title{
Diacronie
}

\section{2/}

\section{Monfalcone, storia di un cantiere navale}

\author{
Stefania Elena CARNEMOLLA*
}

Le navi continuano a essere costruite, a Monfalcone, nel grande stabilimento che guarda verso Trieste. Sono ormai trascorsi più di cento anni da quando la famiglia Cosulich, originaria dell'isola di Lussino, nel golfo del Quarnaro, vi avviò la costruzione di un cantiere dalle alterne vicende e tuttavia destinato a lasciare una traccia nella storia dell'industria navale italiana, grazie, soprattutto, alle navi passeggeri, nonché alle tante unità da carico e militari. Il presente articolo ripercorre le vicende di questo storico cantiere navale, affiancando alla narrazione una ricchissima documentazione fotografica.

\section{La nascita del Cantiere Navale Triestino}

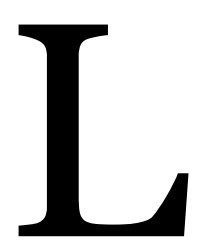

e navi continuano a essere costruite, a Monfalcone, nel grande stabilimento che guarda verso Trieste. Sono ormai trascorsi più di cento anni da quando la famiglia Cosulich, originaria dell'isola di Lussino, nel golfo del Quarnaro, vi avviò la costruzione di un cantiere dalle alterne vicende e tuttavia destinato a lasciare una traccia nella storia dell'industria navale italiana, grazie, soprattutto, alle navi passeggeri, nonché alle tante unità da carico e militari. Ma a Monfalcone sarebbero stati anche costruiti idrovolanti, carrozze ferroviarie, motoscafi, fra cui i Bora, in voga negli anni Sessanta, navi officine semisommergibili, come la Micoperi 70oo, ponti in ferro, come il Polvorilla, pensato per il collegamento fra Cile e Argentina. 
L'arrivo dei Cosulich, Callisto e Alberto, a Monfalcone - ma già attivi a Trieste con l'Unione Austriaca di Navigazione ${ }^{1}$ e, ancora prima, con la F.lli Cosulich - coincise con l'emanazione della legge del 23 febbraio del 1907 per la «sovvenzione della marina mercantile e il promovimento delle costruzioni navali» ${ }^{2}$. Un inedito per la legislazione austriaca in virtù dei contributi di costruzione, che andavano ad affiancarsi a quelli tradizionali di viaggio ed esercizio, ora concessi ai cantieri. Nel momento in cui la crescita dei traffici marittimi continuava a far registrare un incremento della domanda di unità navali, la mossa di Vienna fu interpretata come una misura atta a contenere l'arricchimento dei cantieri e degli armamenti stranieri, il tutto a favore dei corrispondenti comparti nazionali. Gli stessi Cosulich si erano dovuti rivolgere ai cantieri inglesi, considerati all'avanguardia nel settore, né avrebbero potuto fare altrimenti dal momento che i cantieri locali, tranne l'Arsenale del Lloyd Austriaco, si erano rivelati inadeguati per l'allestimento di navi di grosso tonnellaggio, ciò di cui i

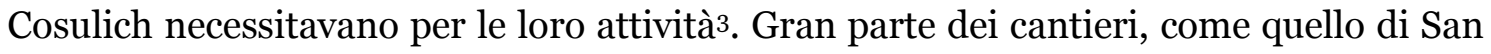
Marco, nato come squero a Trieste nel 1839, erano stati inoltre monopolizzati dalla Marina austro-ungarica. Né il cantiere di San Rocco, nella vicina Muggia, avrebbe potuto sostenere, da solo, l'incremento di commesse di naviglio per l'armamento libero. Davanti alla prospettiva di uno sviluppo delle costruzioni navali nella regione, i Cosulich maturarono pertanto l'idea di un proprio cantiere, ciò che avrebbe consentito loro di ampliare le proprie attività e di affrancarsi gradatamente dai cantieri d'Oltremanica, tanto più che la svalutazione del fiorino austriaco nei confronti della sterlina iniziava a gravare sui tanti acquisti, all'estero, delle navi societarie.

Prima ancora che la nuova disposizione venisse pubblicata sul «Bollettino delle leggi dell'Impero», il 31 gennaio del 1907 «L'Osservatore Triestino» annunciò la costruzione di un cantiere ad opera dei due armatori lussiniani: «Gli effetti della legge concernente la sovvenzione alla marina mercantile ed il promuovimento delle costruzioni navali, testè votata dal Parlamento, si fecero sentire - scrive la Triester Zeitung di ieri - bentosto ed in misura tale quale non se la sarebbe aspettata il migliore degli ottimisti. Astrazion fatta dagli ingrandimenti e dagli ampliamenti dei cantieri che esistono a Trieste, possiamo già oggi dare relazione di un nuovo e grande cantiere, arredato modernamente, che dovrà sorgere a Monfalcone nei pressi delle linee della

\footnotetext{
${ }^{1}$ Nata dalla fusione tra la F.lli Cosulich e la Austro-Americana, società di navigazione dedita ai traffici d'oltremare creata nel 1895 dagli industriali viennesi Schenker e dalla Burrell \& Son di Glasgow, di cui i Cosulich rilevarono le quote.

${ }^{2}$ Bollettino delle leggi dell'Impero per $i$ Regni e Paesi rappresentati nel Consiglio dell'Impero, Vienna, Coi tipi dell'I.R. Stamperia di Corte e di Stato, 1907, pp. 297-302.

$3 \mathrm{Nel} 1900$ i Cosulich avevano inaugurato una regolare linea commerciale con il Nord America e, nel 1904, una per emigranti e passeggeri di classe.
} 
Südbahn o della ferrovia dello Stato. Imprenditori sono i Fratelli Cosulich, di cui sono note le relazioni coll'Austro-Americana e col Bankverein di Vienna, rispettivamente colla Banca commerciale triestina. I passi formali per la nuova impresa sono già avviati e fra giorni verrà presentata all'i.r. Luogotenenza di Trieste la domanda per la concessione della costituzione di una società per azioni con un capitale di 4 milioni di Corone allo scopo d'esercire il cantiere. Oltre a ciò i lavori preliminari pratici sono già al punto che è stato acquistato il fondo necessario e sono state già ordinate alcune macchine, dopo di che inizieranno tosto i lavori di costruzione del cantiere. I fondi confinano col canale navigabile di Monfalcone e col grande bacino annessovi, che recentemente fu scavato e che colla sua estensione di oltre 500.000 metri quadrati offre un posto riparato per lo sviluppo di un importante esercizio di cantiere. Nel cantiere verranno stabiliti, per ora, cinque vasamenti sui quali sarà possibile di costruire contemporaneamente cinque grandi piroscafi da 10.000 tonnellate ciascuno. Oltre a ciò verrà provveduto per la costruzione di macchine, caldaie e di tutti gli altri oggetti d'armo. Il macchinario corrisponderà alle esigenze della tecnica moderna e la forza motrice principale verrà fornita dall'acqua, dall'elettricità e dall'aria compressa. Si ha intenzione di incominciare ancora quest'anno colla costruzione di piroscafi per poterli fornire pronti già nel 1908. Un largo spazio di terreno è riservato per un dock asciutto. I lavori per quest'ultimo non verranno però iniziati tosto, ma si avvieranno dapprima trattative coll'i. e r. Marina da guerra allo scopo di colmare la lacuna di un grande dock asciutto per le navi più grosse della nostra marina da guerra e per i piroscafi da passeggeri. L'esercizio della nuova impresa sarà del tutto separato da quello dell'Austro-Americana. L'impresa conta di potere sviluppare proficuamente la propria attività anzitutto per l'Austro-Americana poi per il Lloyd austriaco e per gli armatori della navigazione libera»4.

Come si arrivò a Monfalcone? Per i lavori di ampliamento del porto di Trieste si erano resi necessari ingenti quantitativi di ghiaia e altrettanti di sabbia che la Pietro Fiaccanoni \& C. era riuscita a reperire nei terreni acquitrinosi della Baia di Panzano, a ovest del porticciolo di Portorosega. I Cosulich, accordatisi con la società, sfruttarono gli scavi per poter ottenere due bacini comunicanti con il mare, provvisti di buoni fondali, distanti pochi chilometri dal centro abitato di Monfalcone e a non più di sedici miglia marittime da Trieste, loro porto d'armamento. L'area di Panzano, costeggiata dal canale navigabile Valentinis, era inoltre alimentata dall'acqua dolce del Canale dei Dottori, l’ideale per immergervi le carene delle navi in allestimento.

\footnotetext{
4 «Un nuovo cantiere austriaco», in L'Osservatore Triestino, 31 gennaio 1907, p. 2. 
Il 3 aprile del 1908 fu costituita la società per azioni Cantiere Navale Triestino presieduta da Alberto e Callisto Cosulich. Li affiancò Arthur Kuffler, presidente dell'associazione degli industriali cotonieri austriaci e in stretti legami con il CreditAnstalt, potente banca austriaca. Estimatori dei metodi dell'industria navale britannica, i Cosulich chiamarono alla guida del Cantiere l’ingegnere scozzese James Stewart, già ispettore tecnico dell'Unione Austriaca di Navigazione, e, in qualità di ingegnere capo, Andrew Munroe. Dal Regno Unito giunsero anche duecento operai specializzati per le varie categorie del gruppo ferro, mentre per la carpenteria in legno Stewart si rivolse a maestri d'ascia lussiniani e istriani. Ribattini, criccatori e addetti alla manovalanza furono invece reclutati tra la gente del Carso e i contadini dell'istriano.

\section{Le prime unità passeggeri}

Fra la primavera e l'estate del 1908 furono impostati sugli scali il Trieste e lo Split, due piroscafi misti di 896 tsl ordinati al Cantiere dalla Società Anonima di Navigazione a Vapore Dalmatia.

Nel 1909 fu la volta del Nereide, piroscafo da carico di 3.405 tsl per la Tommaso Cossovich Società Anonima di Navigazione a Vapore di Trieste.

Nel 1910 i Cosulich ottennero da Vienna una convenzione di sovvenzione statale per la linea di navigazione con l'Argentina. Fu in questo contesto che nacque il Kaiser Franz Joseph I, piroscafo passeggeri di 12.567 tsl per l'Unione Austriaca di Navigazione. Dalla linea esterna tradizionale ed elegante, prua a tagliamare, leggermente inclinata, poppa ellittica, cassero centrale sormontato da due grandi fumaioli, successivamente abbassati, il Kaiser Franz Joseph I, a tre classi, fu varato il 9 settembre del 1911 alla presenza di Maria Gioseffa e Massimiliano, arciduchi d'Austria, compiendo il suo viaggio inaugurale da Trieste a New York il 9 maggio del 1912. Il Titanic, transatlantico della White Star Line, anch'esso diretto a New York, era naufragato solo tre settimane prima.

Il piroscafo passeggeri Kaiserin Elizabeth, impostato il 30 novembre del 1912, con le sue 14.700 tsl sarebbe stato fra i maggiori della marineria asburgica se un incendio non lo avesse danneggiato o se i bombardamenti su Monfalcone del maggio del 1915 non ne avessero compromesso la struttura.

Il 22 agosto del 1913 fu consegnato all'Unione Austriaca di Navigazione il Belvedere, piroscafo misto di 7.166 tsl per il trasporto di merci ed emigranti fra l'Adriatico e il Nord e Sud America, più volte aggiornato e ristrutturato, a partire dagli anni Venti, allorquando le caldaie a vapore furono trasformate per far posto alla 
combustione a nafta, gli spazi passeggeri ridotti e le stive dotate di impianto frigorifero per il trasporto di carne congelata dall'Argentina.

Per il resto, nel primo decennio di vita del Cantiere la produzione destinata al trasporto passeggeri si limitò a due piroscafi a elica di tipo costiero, il San Marco e il Liqeni, rispettivamente di 276 tsl e 96 tsl, commissionato dalla Società di Navigazione Istria-Trieste, il primo, dalla Società di Navigazione a Vapore del Lloyd Austriaco, il secondo.

\section{Fra la Grande Guerra e la caduta di Wall Street}

James Stewart fu rimpatriato nel 1914, poco prima del grande conflitto mondiale. Per Monfalcone non furono anni facili. Così come non lo furono per i Cosulich. Né per il Cantiere. Distrutto dai bombardamenti, lo si ricostruì. La produzione ripartì, ma quando, davanti a un brusco calo nella domanda di naviglio, essa dovette fare i conti con la crisi delle commesse, fu allora che si pensò a una riorganizzazione interna con la creazione delle Officine Ferroviarie, nel 1921, di quelle Elettromeccaniche, nel 1922, delle Officine Aeronautiche, nel 1923. Intanto, nel 1919, l'Unione Austriaca di Navigazione aveva, sotto la direzione generale di Alberto Cosulich e quella tecnicocommerciale di Antonio Nicolò, secondogenito di Callisto, cambiato la propria ragione sociale in quella di Cosulich Società Triestina di Navigazione. Seppure su scala ridotta, la nuova società aveva riaperto i servizi commerciali e le rotte passeggeri per gli Stati Uniti e il Sud America. Anche il Kaiser Franz Joseph I aveva ripreso il mare, benché con il nome di Presidente Wilson. Negli anni Venti la produzione fu in linea di massima caratterizzata dalla costruzione di bacini galleggianti, chiatte, motonavi miste e da carico, motoscafi passeggeri, piroscafi cisterna e da carico, posamine, rimorchiatori, sommergibili. Né vennero a mancare unità di pregio, come il mercantile Mauly, il piroscafo cisterna Dora e il sommergibile Vettor Pisani, consegnato alla Regia Marina il 15 giugno del 1929.

Nel 1929 l'onda lunga del crollo di Wall Street investì l'Europa, abbattendosi anche sull'industria monfalconese, già messa a dura prova dalla struttura del mercato italiano e dalla organizzazione interna dei principali nuclei produttivi. Anche la società dei Cosulich conobbe le sue traversie. Con il fallimento della Banca Commerciale Triestina, che fino ad allora aveva contribuito a reggerne le sorti, la maggioranza delle azioni furono rilevate dalla Banca Commerciale Italiana, insediatasi a Trieste sin dal dopoguerra. Ciò condusse, il 6 novembre del 1929, a un riordino della dirigenza della Cosulich Società Triestina di Navigazione. Assicuratosi il controllo del Cantiere Navale 
Triestino, nonché quello dei cantieri di Muggia e Trieste, l'istituto milanese riorganizzò il settore, dando vita, nel 1930, alla società anonima per azioni Cantieri Riuniti dell'Adriatico.

\section{Saturnia e Vulcania, due gioielli degli anni Venti}

Vi è la firma dell'architetto Arduino Berlam e Nicolò Costanzi, carenista, designer d'esterni, abile pianificatore di spazi interni, sulla Saturnia e la Vulcania, due motonavi passeggeri ordinate al Cantiere dai Cosulich prima ancora che traversie societarie e problemi finanziari intervenissero sulle loro vicende imprenditoriali. La Saturnia compì il suo viaggio inaugurale il 21 settembre del 1927, diretta a Buenos Aires (sua madrina di varo, il 29 dicembre del 1925, fu la Principessa Giovanna di Savoia), la Vulcania, il 19 dicembre del 1928, destinazione New York.

Tradizione vuole che in vista della realizzazione della Saturnia, Costanzi, futuro capo dell'Ufficio Tecnico del Cantiere, raggiungesse l'Inghilterra su invito dei Cosulich per acquisire la necessaria esperienza. Probabile, invece, vi arrivasse con i rotoli dei bozzetti sottobraccio. Benché la Saturnia e la Vulcania finissero col presentare elementi tradizionali tipicamente inglesi tanto nello scafo quanto nei castelli dei ponti, esse risentirono della mano sperimentatrice di Costanzi. Delle due unità, dalla poppa ellittica, prua leggermente inclinata in avanti, i due alberi perpendicolari, si concentrò soprattutto sul cassero centrale, a quattro ponti, sovrastato da un solo fumaiolo basso e largo di forma troncoconica. Ciò influì sulla loro sagoma, secondo un sistema compositivo destinato a fungere da archetipo di riferimento per le costruzioni passeggeri italiane del secondo dopoguerra. L'aggiornamento riguardò anche le carene, attraverso l'adozione del cutaway on the forefoot e dello overhang. Costanzi procedette, cioè, a un rialzo della parte anteriore della chiglia verso prua in modo da facilitare la penetrazione dello scafo nel mezzo liquido grazie alla diminuzione della superficie bagnata dell'opera viva, quindi a un allungamento a guisa di pinna a poppavia all'altezza delle linee d'acqua, secondo una soluzione comune ai più veloci transatlantici inglesi.

L'apparato motore, costruito dalla Fabbrica Macchine Sant'Andrea dello Stabilimento Tecnico Triestino, comprendeva, oltre agli ausiliari e alla centrale Diesel elettrica, due motori di propulsione a combustione interna tipo Burmeister \& Wain capaci di assicurare una velocità di crociera di 20 nodi.

La Prima Classe fu progettata per poter accogliere ampi e lussuosi saloni, passeggiate coperte e all'aperto, una grande piscina coperta con lido e servizi, cabine 
con veranda esterna complete di servizi igienici privati. Più sobrio, benché decoroso, l'arredamento delle altre classi, a loro volta vivacizzate da sale di ritrovo e passeggiate all'aperto.

\section{Gli anni Trenta}

Nel 1932 nacque la Società Italia-Flotte Riunite, frutto della razionalizzazione del comparto marittimo, resasi necessaria davanti all'impossibilità, da parte dello Stato, di continuare a sovvenzionare sprechi ed errori degli operatori del settore. Nella nuova società confluirono il Lloyd Sabaudo, la Navigazione Generale Italiana, la Cosulich Società Triestina di Navigazione. Nel 1937 queste furono assorbite, insieme a Italia Navigazione, Lloyd Triestino, Adriatica di Navigazione, Tirrenia di Navigazione, nella Finmare, holding finanziaria dell'Istituto per la Ricostruzione Industriale. Fondato nel gennaio del 1933, auspice il clima di risanamento del tessuto industriale, economico, bancario, finaziario del paese, l'IRI aveva a sua volta rilevato le partecipazioni industriali della Banca Commerciale Italiana, assumendo, contestualmente, il controllo dei Cantieri Riuniti dell'Adriatico.

Negli anni Trenta il Cantiere di Monfalcone divenne leader europeo nella costruzione di sommergibili, navi mercantili e passeggeri. Fu, questa, l'epoca delle grandi motonavi Neptunia, Oceania, Pilsudski, Batory, Stockholm, nonché della piccola Calitea, motonave mista di 4.013 tsl, a quattro stive, due eliche, sistemazioni per passeggeri di classe, arredamenti eleganti e razionali, concepita per la linea del Levante e del Dodecaneso.

La Neptunia di 19.327 tsl, varata nel 1931, fu consegnata alla Cosulich Società Triestina di Navigazione il 30 settembre del 1932 per i viaggi lungo la rotta del Sud America. Dalla sagoma elegante e slanciata, simile a quella della Saturnia, essa si caratterizzò tuttavia per un'eleganza più sobria oltre che per l'articolazione in due sole classi, Unica e Turistica, pur con il mantenimento di ampi spazi destinati allo svago e al ristoro.

Nell'estate del 1933 la Oceania di 19.403 tsl, ordinata al Cantiere dalla società di navigazione dei Cosulich, compì alcune crociere attraverso il Mediterraneo prima di salpare l'àncora da Trieste diretta a Buenos Aires.

La Pilsudski di 14.923 tsl fu invece realizzata per il trasporto passeggeri fra i porti polacchi e il Nord America. Varata il 19 dicembre del 1934, fu consegnata alla Gydnia America Line di Varsavia il 13 agosto del 1935. Dalla linea elegante, prua leggermente inclinata, poppa tipo incrociatore, presentava sovrastrutture continue sormontate da 
due fumaioli a sezione elissoidale. La gemella Batory, di pari tonnellate di stazza lorda e identiche caratteristiche tecnico-alberghiere, fu varata il 3 luglio del 1935. Consegnata all'armatore il 18 aprile del 1936, partì per New York il 18 maggio di quell'anno. Tanto la Pilsudski quanto la Batory furono costruite a sèguito di un accordo fra il governo italiano e quello polacco per la fornitura di carbone alle industrie nazionali. E con il carbone le si pagò.

Simbolo degli anni Trenta, con i suoi dieci ponti, prua slanciata, poppa tipo incrociatore, sovrastrutture sormontate da due fumaioli di sezione ellittica, fu la motonave Stockholm, transatlantico di lusso di 28.000 tsl ordinato ai Cantieri Riuniti dell'Adriatico dalla Svenska Amerika Linie di Göteborg per la linea celere fra la Scandinavia e il Nord America. Impostata il 10 aprile del 1937, varata il 29 maggio del 1938, un incendio del 19 dicembre di quell'anno ne demolì sovrastrutture e parte dello scafo. Quanto rimasto dell'opera viva fu arripato sullo scalo perché si procedesse ai lavori di ristrutturazione. L'unità fu varata, in forma strettamente privata, il 10 marzo del 1940. Nonostante l'ingresso dell'Italia in guerra, ciò non ne ostacolò l'allestimento. Nel giugno del 1941 scese in mare dalle parti di Fiume. Inviata a Venezia, fu sottoposta ad alcuni interventi alla carena. Rifiutata in tempo di guerra dal primo armatore, fu acquistata dalla Italia Società Anonima di Navigazione di Genova, che la ribattezzò con il nome di Sabaudia. Dopo l'8 settembre del 1943 fu trasferita dall'occupante tedesco nel Vallone di Zaule, dove affondò, colpita da aerei inglesi, il 6 luglio del 1944. La Stockholm poi Sabaudia fu per gli anni Trenta quello che la Saturnia e la Vulcania erano state per gli anni Venti, esempio di eleganza, innovazione, ricerca continua di potenza. Era stata progettata con sovrastrutture in acciaio ad alta resistenza tanto da rendere necessario l'impiego della saldatura elettrica in luogo della tradizionale chiodatura, con tre motori di propulsione tipo CRDA-Sulzer perché agissero su tre eliche, nonché, elemento di novità, con impianto d'aria condizionata per il sollievo dei passeggeri.

\section{Ferite di guerra}

Nel 1939 la Finmare approvò un programma di ammodernamento della propria flotta. Rientrò in questo contesto la Calino, motonave mista di 5.185 tsl ordinata dalla Adriatica Società Anonima di Navigazione di Venezia per i servizi sovvenzionati con l'Egitto e il Levante. Con buona capacità di stiva, ottime sistemazioni passeggeri, arredata con lusso e razionalità, fu varata il 12 agosto del 1939. Tenuta in disarmo a Venezia, fu requisita dalla Regia Marina. Trasferita a Napoli, il 5 novembre del 1940 
partì in convoglio alla volta di Tripoli, Bengasi, Tobruk per una prima missione di guerra. Affondò il 19 gennaio del 1943 a seguito dell'urto contro una mina ancorata al largo di Punta Campanella, lungo la costa tirrenica, vanificando i soccorsi del cacciatorpediniere Vincenzo Gioberti, a cui tuttavia riuscì di mettere in salvo alcuni naufraghi.

La Andrea Gritti con le sue 6.405 tsl era stata progettata come motonave da carico per la Società Italiana d'Armamento di Fiume. Consegnata nel luglio del 1943, fu tuttavia requisita dalla Regia Marina. Restituita nell'agosto del 1945, fu adattata al trasporto di emigranti. Compì il suo viaggio inaugurale da Genova a Buenos Aires il 12 agosto del 1946. Fu, la sua, la prima partenza di una nave passeggeri italiana nel dopoguerra.

Il 20 ottobre del 1941 fu varata la motonave passeggeri Ausonia destinata alla flotta della Adriatica Società Anonima di Navigazione di Venezia in sostituzione di una turbonave dello stesso nome incendiatasi nel 1935 nel porto di Alessandria d'Egitto. La nuova Ausonia non ebbe miglior sorte. Rinacque come Esperia nel 1949 dopo essere rimasta rovesciata su un fianco all'interno del Cantiere. Di 9.314 tsl, sobria ed elegante al tempo stesso, dalla prua slanciata, poppa tipo incrociatore, sovrastrutture centrali sormontate da un solo fumaiolo, disponeva di sette ponti, di cui due continui, quattro stive e sistemazioni passeggeri suddivise in tre classi, con la possibilità, per il cabotaggio fra Alessandria d'Egitto e Porto Said, di imbarcare quarantaquattro passeggeri da ponte. L'apparato motore era composto, oltre che dai necessari ausiliari, da due motori tipo CRDA-Sulzer, di dieci cilindri ciascuno, nonché da cinque elettrogeni da $225 \mathrm{KW}$. Fu inoltre dotata di moderni strumenti di navigazione come radar, radiogoniometro, girobussola Sperry, scandaglio a ultrasuoni. E, fra la stiva due e i locali ausiliari, per il diletto degli ospiti, nonché per la prima volta nella storia di una nave passeggeri italiana, di una sala cinematografica da centoventicinque posti. Una novità riguardò anche il Ponte Sole, con la creazione di un sistema di giochi d'acqua all'interno di due padiglioni di vetro. Completavano l'attrezzatura del Lido, un bar con terrazzo, tende, ombrelloni, spazi riservati ai bagni di sole.

Per far fronte all'elevata richiesta di unità per emigranti, alla fine degli anni Quaranta fu costruita la motonave mista Francesco Morosini di 8.525 tsl. Varata il 12 dicembre del 1947, consegnata alla Società Italiana d'Armamento di Venezia il 31 maggio del 1948, compì il suo viaggio inaugurale il 19 giugno di quell'anno, diretta a Buenos Aires. Benché concepita per passeggeri di Classe Economica fu allestita con decoro, laddove la si dotò di buoni impianti igienico-sanitari, sala da pranzo, veranda, bar, piscina. 
Il Cantiere fu distrutto dai bombardamenti del 1944. Grazie all'intervento dell'IRI e del Governo Militare Alleato affrontò la seconda ricostruzione della sua storia.

Nel 1948 giunse a Monfalcone, quasi irriconoscibile - dopo aver servito con il nome di Hermitage come nave per il trasporto truppe della U.S. Navy - il Conte Biancamano, glorioso transatlantico degli anni Venti costruito dal cantiere W. Beardmore \& Co. Ltd. di Dalmuir per il Lloyd Sabaudo di Genova. Completati i lavori di ammodernamento, il 26 ottobre del 1949 fu dato in gestione alla Società Marittima Nazionale di Roma. Venduto nel 1952 alla Italia S.p.A. di Navigazione di Genova per il trasporto passeggeri lungo la rotta del Nord e Sud America, il 16 agosto del 1960 fu demolito a La Spezia dalla Terrestre Marittima.

\section{La Giulio Cesare e il ritorno delle grandi navi passeggeri}

La motonave passeggeri Giulio Cesare di 27.227 tsl dagli ampi saloni, arredamento razionale ed elegante, luminose passeggiate, spazi all'aperto destinati al gioco e al relax, fu varata il 18 maggio del 1950 per la Italia S.p.A. di Navigazione di Genova. Dopo una crociera attraverso il Mediterraneo, compì il suo viaggio inaugurale il 27 ottobre del 1951, diretta a Buenos Aires. Durante le prime prove in mare, i suoi quattro motori FIAT, riesumati dall'archivio prebellico, le avevano permesso di raggiungere una velocità di crociera di 23,2 nodi. Un risultato notevole, per l'epoca. Ma fu soprattutto l'aspetto esterno a differenziare la Giulio Cesare, primo transatlantico italiano del dopoguerra, da tutti gli omologhi sino ad allora costruiti. La prua, slanciata, a tagliamare, la poppa, tondeggiante, tipo incrociatore, le sovrastrutture, degradanti verso poppa, sormontate da un unico albero sagomato e da un grande fumaiolo dalla linea leggermente aerodinamica, tutto ciò le conferiva l'eleganza di un panfilo.

La motonave mista Africa, di 11.427 tsl, fu richiesta ai Cantieri Riuniti dell'Adriatico dal Lloyd Triestino S.p.A. di Navigazione per la linea del Sud Africa. Con buona capacità di stiva, di eleganza sobria, prua slanciata, poppa tipo incrociatore, sovrastrutture centrali molto avviate, un solo fumaiolo sagomato e albero a tripode, fu assai attiva lungo la rotta che le era stata predestinata, poi dirottata via Gibilterra nel 1956 e nel 1967 a sèguito della prima e seconda chiusura del Canale di Suez.

Il 20 settembre del 1957 fu consegnata alla Adriatica S.p.A. di Navigazione di Venezia la Ausonia, turbonave passeggeri di gran lusso di 11.879 tsl progettata per il Grande Espresso Italia-Egitto-Libano. Dalla prua fortemente inclinata, poppa tipo incrociatore, un solo fumaiolo dalla linea aerodinamica a sezione elissoidale, albero prodiero a tripode, sovrastruttura completa con cassero e tuga centrale, fu la prima tra 
le navi passeggeri italiane a montare un impianto di stabilizzatori antirollio a pinne rientranti e orientabili tipo Denny Brown. Sul Ponte Lido, accanto alla zona piscina, a sua volta protetta da una vetrata paravento e cupola, rimovibile, in materiale plastico, fu invece installato un sistema di riscaldamento a raggi infrarossi per la fruizione di tale spazio anche in condizioni climatiche proibitive.

Stabilizzatori antirollio furono adottati anche sulla Stelvio, motonave mista di 4.408 tsl varata il 6 luglio del 1958 e costruita per la Adriatica S.p.A. di Navigazione di Venezia per i viaggi in classe unica lungo la rotta per la Grecia e i porti dell'Egeo.

Nel 1954 fu trasferita a Monfalcone per lavori di ammodernamento la Homeric, turbonave a due eliche per i servizi transpacifici di classe varata il 18 luglio del 1931 nel Cantiere di Quincy della Bethlehem Shipbuilding Corporation, cui era stata ordinata dalla Matson Navigation Company di San Francisco. Requisita in tempo di guerra dalla U.S. Navy per il trasporto truppe, nel 1953 passò alla Home Lines Inc. di Panama dopo lungo disarmo nel porto di Alameda. Uscì da Monfalcone rinnovata esternamente, con l'applicazione di una nuova prua nella zona dell'opera morta, ma anche internamente, con la completa revisione degli spazi destinati ai passeggeri.

Nel 1959 l'IRI annunciò la nascita di una propria finanziaria perché rilevasse le partecipazioni delle società facenti capo al comparto delle riparazioni e costruzioni navali per poterne assumere il coordinamento tecnico e finanziario. Era la Fincantieri.

\section{Le ultime regine del mare}

Negli anni Sessanta la scienza italiana fu celebrata attraverso due grandi navi, fra le più imponenti costruite a Monfalcone, in pieno boom economico: la Galileo Galilei e la Guglielmo Marconi, rispettivamente di 27.866 tsl e 27.905 tsl e ordinate ai Cantieri Riuniti dell'Adriatico dal Lloyd Triestino S.p.A. di Navigazione. Di certo non spiccarono per sfarzo, cui fu invece preferito un garbato decoro, Il primato riguardò ben altro. L'ingegneria navale stava facendo passi da gigante. Per l'epoca e il contesto. La Galileo Galilei, considerata all'avanguardia per le sue caratteristiche tecniche, fu ad esempio insignita del Premio ANIAI, riconoscimento dell'Associazione Nazionale Ingegneri Architetti Italiani, con la seguente motivazione: «La T/n Galileo Galilei, del Lloyd Triestino, costruita dai Cantieri Riuniti dell'Adriatico, fra le grandi navi di linea realizzate in Italia nell'ultimo settennio è quella che ha richiamato in modo particolare la attenzione della Commissione, non tanto per le sue caratteristiche di funzionalità e di tradizionale buon gusto, che hanno fatto di questa nave uno dei più ambiti mezzi di trasporto sulla Rotta dell'Estremo Oriente e dell'Australia, quanto per alcune 
caratteristiche tecniche, di indiscussa originalità, che hanno assicurato alla nave valori di efficienza mai raggiunti contemporaneamente in precedenti opere navali mercantili». Quali? «La cosiddetta sede d'onda» precisava la motivazione «introdotta nella parte prodiera della carena, il rigonfio centrale dello scafo a guisa di controcarena subacquea, sono originali innovazioni tecniche che hanno consentito di ridurre considerevolmente la resistenza al moto e di incrementare la stabilità per le condizioni di nave scarica o semiscarica, con conseguente eliminazione dello zavorramento permanente: risultati tecnici che si traducono in importanti benefici economici di esercizio» 5 .

La Guglielmo Marconi ne condivise caratteristiche tecnico-alberghiere. Comune alle due unità fu anche l'apparato motore composto da tre caldaie tipo CRDA-Barcock \& Wilcox, due gruppi turboriduttori tipo CRDA-De Laval, realizzati dalla Fabbrica Macchine Sant'Andrea, nonché dagli ausiliari per i servizi di macchina e di bordo.

Il 15 gennaio del 1963 fu varata la Oceanic, turbonave passeggeri di 39.241 tsl ordinata ai Cantieri Riuniti dell'Adriatico dalla Home Line Inc. di Panama. Transatlantico di lusso dalla linea esterna alquanto elegante, con prua a clipper e sede d'onda, poppa tipo incrociatore assai slanciata, sovrastrutture continue sormontate da un albero carenato a prua e da un elegante fumaiolo a poppa, dotata di stabilizzatori tipo Denny Brown, il 24 aprile del 1965 la Oceanic partì per il suo viaggio inaugurale da Trieste, diretta a New York e da qui alle Bahamas.

La Eugenio C. turbonave passeggeri di 30.567 tsl costruita per la Costa Armatori S.p.A. di Genova fu l'ultima delle grandi navi. Confortevole e sicura, realizzata con arredamenti d'alta classe, ampi saloni, verande luminose, spazi per attività ludiche e piscine all'aperto, provvista di stabilizzatori tipo Denny Brown, nonché dei più moderni ritrovati tecnici, varata il 21 novembre del 1964, partì da Genova per il suo viaggio inaugurale il 22 agosto del 1966, diretta a Buenos Aires, realizzando la sua prima traversata alla velocità di 27,5 nodi. Rispetto a tutte le altre unità entrate in servizio fino a quel momento, la Eugenio $C$. si distinse per la sistemazione dell'apparato motore nella zona poppiera e per due fumaioli appaiati dalla forma aerodinamica e originale studiata per ridurre al minimo il fastidio della ricaduta sui ponti dei prodotti di combustione. Lo scafo invece ricalcò la linea iniziata dai Cantieri Riuniti dell'Adriatico sui tipi Galilei e confermata sulla Oceanic, con prua a cutter e poppa molto slanciata di tipo whale boat, con riquadro sotto la linea di galleggiamento atto a ridurre l'onda di poppa e a migliorare il rendimento della carena.

5 In cantiere. Tecnica, arte, lavoro. Ottant'anni di attività dello stabilimento di Monfalcone, a cura di Valerio Staccioli, Monfalcone, Edizioni della Laguna, 1988, p. 271. 
Il 1966 segnò la confluenza dei Cantieri Riuniti dell'Adriatico e della Ansaldo, le due maggiori realtà cantieristiche del paese, nella Italcantieri, nuova società del gruppo IRI. Da quel momento il Cantiere di Monfalcone si orientò verso la costruzione di navi cisterna, motoscafi Bora, unità militari.

Il 30 giugno del 1984 la Fincantieri fu trasformata in società operativa, con sede a Trieste, assumendo la ragione sociale di Fincantieri Cantieri Navali S.p.A. Nel 1985 incorporò la Italcantieri, la Cantieri Navali Riuniti - cui facevano capo gli stabilimenti di Genova, Muggiano, Riva Trigoso, Genova, Palermo - la Grandi Motori Trieste, il Cantiere Navale Breda di Venezia Marghera, l'Arsenale Triestino San Marco, i Cantieri Navali e le Officine Meccaniche di Venezia, il Cantiere Navale Luigi Orlando di Livorno, la Società per l'Esercizio dei Bacini Meridionali di Napoli. Fu, per la cantieristica del paese, l’inizio di un'importante fase di riorganizzazione, risanamento, rilancio. Sarà in questo rinnovato contesto che il cantiere di Monfalcone farà del ritorno alla costruzione delle navi passeggeri e anni dopo la partenza della Eugenio $C$. il punto di forza della sua nuova storia, rientrando in un mercato di nicchia come poteva essere allora quello dei cruise liners, al riparo dalla concorrenza dei cantieri dell'Estremo Oriente, privi del know-how necessario nel campo delle costruzioni navali ad alto contenuto tecnologico.

\section{L'ultimo sommergibile saluta Monfalcone}

Il 26 giugno del 1993 il sommergibile Gianfranco Gazzana Priaroggia scese in mare dallo scalo gigante, salutato dal suono delle sirene. Sarebbe stato l'ultimo sommergibile varato a Monfalcone, nonché l'ultimo varo con scivolamento in mare, ottantacinque anni dopo quello del piroscafo Trieste. Quel giugno del 1993, a fare da cornice, ormeggiata alla banchina d'allestimento E, la nave da crociera Maasdam. La piccola unità della Marina Militare quasi sembrò scomparire di fronte al grande scafo blu scuro della nave mentre le passava accanto trainata dai rimorchiatori. Fu quella, per Monfalcone, una giornata storica, di passaggio. Da un'epoca all'altra. Oggi, dove un tempo nascevano grandi transatlantici, si costruiscono navi da crociera per i maggiori marchi dell'armamento mondiale 


\section{BIBLIOGRAFIA}

MARTINUZZI, Matteo (a cura di), Cantiere 100 Anni di navi a Monfalcone, San Dorligo della Valle, Graphart Trieste, 2008.

STACCIOLI, Valerio (a cura di), In cantiere. Tecnica, arte, lavoro. Ottant'anni di attività dello stabilimento di Monfalcone, Monfalcone, Edizioni della Laguna, 1988.

CARNEMOLLA, Stefania Elena, «Da Lussino a Monfalcone. Dai Cosulich a Fincantieri: le navi passeggeri nella storia della cantieristica italiana», in Rivista Marittima, Agosto/Settembre 2008, pp. 65-84.

FRAGIACOMO, Paolo, La grande fabbrica, la piccola città. Monfalcone e il cantiere navale: la nascita di una company town: 1860-1940, Milano, Franco Angeli, 1997.

PERUZZI, Luca, «Sommergibili, tecnologia e cantieristica dal 1907 al 2007», in Rivista Marittima, Marzo 2008, pp. 51-6o.

TURRINI, Alessandro, Gli squali dell'Adriatico. Monfalcone e $i$ suoi sommergibili nella storia navale italiana, Gorizia, Vittorelli Edizioni, 1999.

VALENTI, Paolo, Storia del cantiere navale di Monfalcone. 1908-20o8, Trieste, Edizioni Luglio, 2007. 


\section{* L'autore}

Laureatasi presso l'Università degli Studi di Pisa con una tesi sulla storia dell'espansione portoghese in Asia, è stata borsista della "Fundação Calouste Gulbenkian" di Lisbona e di altre istituzioni portoghesi, occupandosi in questa veste di storia dei rapporti fra Portogallo e realtà extraeuropee. Autrice di saggi e pubblicazioni, dal 2005 è anche giornalista pubblicista. Nel 2008 un suo servizio ha ricevuto una menzione speciale al "Premio Internazionale di Giornalismo della Repubblica di San Marino”. Nel 2009 una sua prima inchiesta sui crimini nazisti di Bielorussia nei confronti prigionieri di guerra italiani ha ricevuto menzione al "Premio Auschwitz". Nell'ottobre del 2011 è stata nominata advisor della "European Holocaust Research Infrastructure" collegata allo "Institute for War, Holocaust and Genocide Studies" della Royal Academy of Arts and Science di Amsterdam. Da tempo si occupa di crimini nazisti in Bielorussia contro prigionieri di guerra italiani, non ultimo attraverso l'analisi dei documenti del KGB bielorusso.

URL: < http://www.studistorici.com/progett/autori/\#Carnemolla >

\section{Per citare questo articolo:}

CARNEMOLLA, Stefania Elena, «Monfalcone, storia di un cantiere navale», Diacronie. Studi di Storia Contemporanea: Sulle tracce delle idee, 29/12/2012,

URL:< http://www.studistorici.com/2012/12/29/carnemolla_numero_12/ >

Diacronie Studi di Storia Contemporanea $\beta$ www.diacronie.it

Risorsa digitale indipendente a carattere storiografico. Uscita trimestrale. redazione.diacronie@hotmail.it

Comitato di redazione: Marco Abram - Jacopo Bassi - Luca Bufarale - Gianluca Canè - Alessandro Cattunar - Alice De Rensis - Barbara Galimberti - Deborah Paci - Fausto Pietrancosta - Matteo Tomasoni - Luca Zuccolo

Diritti: gli articoli di Diacronie. Studi di Storia Contemporanea sono pubblicati sotto licenza Creative Commons 2.5 . Possono essere riprodotti a patto di non modificarne i contenuti e di non usarli per fini commerciali. La citazione di estratti è comunque sempre autorizzata, nei limiti previsti dalla legge. 